\title{
Motorised momentum exchange space tethers: the dynamics of asymmetrical tethers, and some recent new applications
}

\author{
Matthew Cartmell ${ }^{1,2, *}$, Olga Ganilova ${ }^{1,2}$, Eoin Lennon ${ }^{1}$, and Gavin Shuttleworth ${ }^{1}$ \\ ${ }^{1}$ Department of Mechanical \& Aerospace Engineering, University of Strathclyde, Glasgow, G1 1XJ, Scotland, UK \\ ${ }^{2}$ Strathclyde Space Institute, University of Strathclyde, Glasgow, G1 1XJ, Scotland, UK
}

\begin{abstract}
This paper reports on a first attempt to model the dynamics of an asymmetrical motorised momentum exchange tether for spacecraft payload propulsion, and it also provides some interesting summary results for two novel applications for motorised momentum exchange tethers. The asymmetrical tether analysis is very important because it represents the problematic scenario when payload mass unbalance intrudes, due to unexpected payload loss or failure to retrieve. Mass symmetry is highly desirable both dynamically and logistically, but it is shown in this paper that there is still realistic potential for mission rescue should an asymmetry condition arise. Conceptual designs for tethered payload release from LEO and lunar tether delivery and retrieval are also presented as options for future development.
\end{abstract}

\section{Introduction}

Momentum exchange tether dynamics have been extensively studied in recent years, and are of current considerable interest internationally as a technology for cost effective and environmentally clean propulsion of payload mass from Low Earth Orbit (LEO). A conceptual design for a motorised momentum exchange tether is given in Figure 1 where the central facility motor drive shaft attaches to the propulsion sub-spans by mean of a gantry, mounted so that it can be rotated by the shaft. Typical performance figures for an operational motorised MET on a circular LEO predict an orbit velocity in the region of $7.6 \mathrm{~km} / \mathrm{s}$ and a tether generated increment of around $3.1 \mathrm{~km} / \mathrm{s}$, which has the potential to accelerate the outer payload, at an optimal release position, to around $10.7 \mathrm{~km} / \mathrm{s}$. This is fast enough for Earth escape, using a tether made of Spectra $2000^{\mathrm{TM}}$. It should be noted that a fundamental requirement for motorised spin is shown in Figure 1 comprising a counter-inertia in the form of an additional pair of tethers and counter-masses attached to the motor stator [1]. This sub-system is required to satisfy Newton's third law of motion and is not analysed here but is assumed to be present, coupling through the drive motor electrodynamics in order to provide the requisite operational configuration.

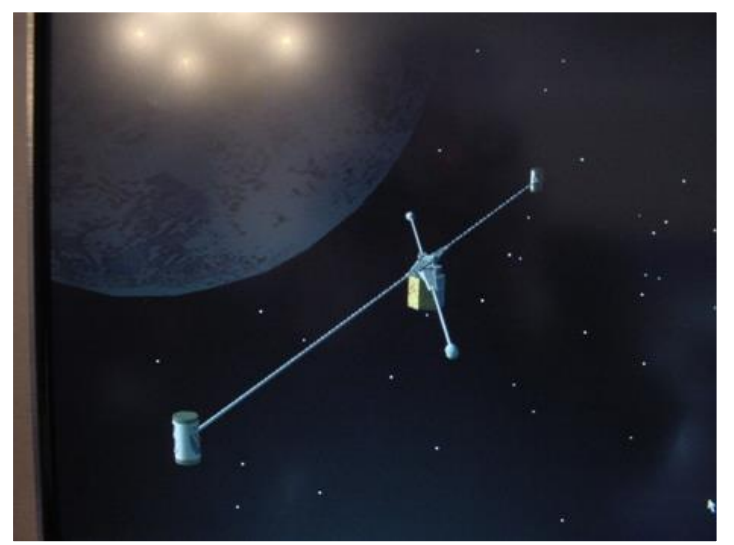

Fig. 1. A motorised momentum exchange tether in LEO.

A fairly comprehensive review of space tether research up to 2008 is given in [2] and a detailed study of fundamental rigid-body motorised tether system dynamics is given in $[3,4]$. Tether flexure, accommodating various forms of vibration, is discussed in full in [5]. Important mission scenarios for motorised tethers are discussed in $[6,7]$. The fundamental issue with asymmetrical payload mass distribution is that the centre of mass location of the tether will then change on orbit, introducing dynamic perturbations that will de-orbit the tether, with potentially disastrous consequences for the mission. In order to maintain the orbit, and also to capitalise on opportunities offered by the exploitation of orbital harmonics, payload mass symmetry is required. In terms of mission architecture this allows a simultaneous in and out flow of payload from the location of the host planet to the destination planet [6]. On the basis that symmetry (defined as the tether being laden with two payloads of identical mass properties 
located at both ends, or, conversely, no payloads on either end of the tether) cannot be absolutely guaranteed, a study of the asymmetrical mass distribution problem was seen to be necessary. In this paper an initial model is proposed, with some calculations that suggest that a pragmatic level of reserve chemical propulsion on board each payload could be used to rescue a mission in which asymmetry has arisen for some reason. This is investigated in the form of the complete loss of one of the payloads. This is followed by discussions of two further investigations in which the tethered release of small payloads from an Earth orbiter and the use of a specialised lunavator tether with an end reeling facility for lunar touchdown are investigated. These two application scenarios in fact reflect different levels of mass symmetry requirement and on that basis they provide interesting platforms for further research into robust solutions to the tether asymmetry problem.

\section{Reference model for a planar motorised tether on an elliptical Low Earth Orbit}

We start with the kinetic and potential energies for a mass-discretised dumb-bell tether (i.e. in symmetrical rigid-body form), and these can be stated as follows [3] for the system shown diagrammatically in Figure 2, with an Earth-fixed frame EXY and a tether-fixed frame $\mathrm{Cx}_{0} \mathrm{y}_{0}$. The tether is assumed to be symmetrical in length and therefore point $\mathrm{C}$, the location of the central facility, is exactly half-way between each payload, $M_{\mathrm{p}}$. Note that the time argument has been dropped from $R(\mathrm{t}), \theta(\mathrm{t})$, and $\psi(\mathrm{t})$ in both equations for clarity and compactness. Quantities are defined in full after the equations.

$$
\begin{gathered}
T=\left[M_{p}+\rho A l+\frac{M_{m}}{2}\right]\left(\dot{R}^{2}+R^{2} \dot{\theta}^{2}\right) \\
+\left[\begin{array}{c}
M_{p}\left(l^{2}+\frac{r_{p}^{2}}{2}\right)+M_{m} \frac{r_{m}^{2}}{4}+ \\
\frac{\rho A l}{12}\left(4 l^{2}+3 r_{T}^{2}\right)
\end{array}\right](\dot{\psi}+\dot{\theta})^{2} \\
U=-\sum_{j=1}^{n} \frac{A \mu l \rho}{n \sqrt{\frac{(2 j-1)^{2} l^{2}}{4 n^{2}}-\frac{(2 j-1) l R \cos \psi}{n}+R^{2}}}- \\
\sum_{j=1}^{n} \frac{A \mu l \rho}{\sqrt{\frac{(2 j-1)^{2} l^{2}}{4 n^{2}}+\frac{(2 j-1) l R \cos \psi}{n}+R^{2}}}- \\
\frac{\mu M_{p}}{\sqrt{l^{2}-2 l R \cos \psi+R^{2}}}-
\end{gathered}
$$

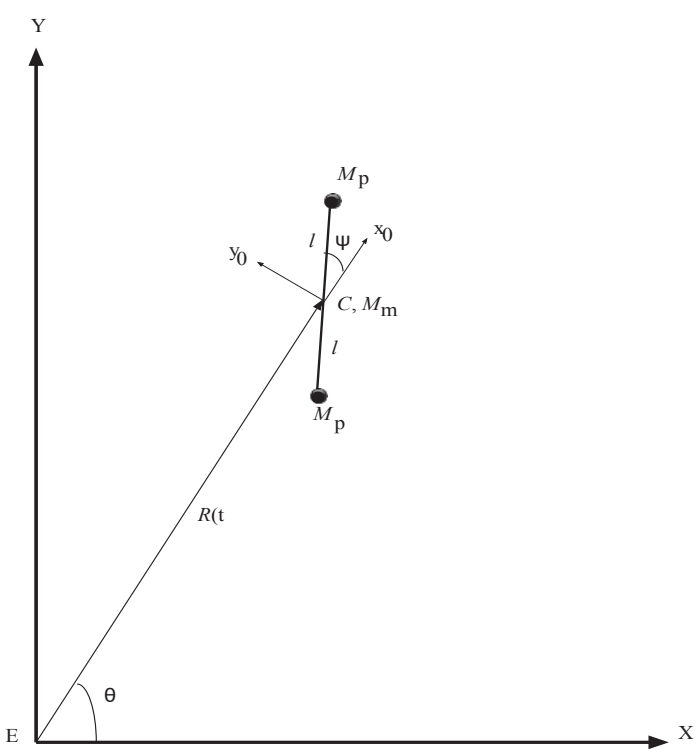

Fig. 2. A planar dumb-bell tether in LEO where E represents the centre of the Earth and EXY is an Earth fixed frame.

Lagrange's equations are used to obtain three differential equations of motion based on generalised coordinates $R(\mathrm{t}), \theta(\mathrm{t})$, and $\psi(\mathrm{t})$. These are intricately coupled, nonlinear, and lengthy, and are not reproduced here but are obtainable in full in [3]. They represent the planar orbital dynamics of the tether, accommodating orbital eccentricity. The data used for the study is as follows. Tether sub-span length $l=50$ $\mathrm{km}$, payload masses $M_{\mathrm{p}}=1000 \mathrm{~kg}$, central facility mass $M_{\mathrm{m}}=5000 \mathrm{~kg}$, tether density $\rho=970 \mathrm{~kg} / \mathrm{m}^{3}$, crosssectional area $A=62.83 * 10^{-6} \mathrm{~m}^{2}$, equal radii of central facility and payloads $r_{\mathrm{m}}=r_{\mathrm{p}}=0.5 \mathrm{~m}$, standard gravitational parameter $\mu=3.9877848 * 10^{14} \mathrm{~m}^{3} / \mathrm{s}^{2}$, drive torque $\tau=5 \mathrm{MNm}$, and integration time $=86400$ s. Initial conditions are $R(0)=6870000 \mathrm{~m}, \theta(0)=$ $97.1877 \mathrm{rad}$, and $\psi(0)=0 \mathrm{rad}$, and $R^{\prime}(0)=0 \mathrm{~m} / \mathrm{s}, \theta^{\prime}(0)$ $=0.00121485 \mathrm{rad} / \mathrm{s}$, and $\psi^{\prime}(0)=0 \mathrm{rad} / \mathrm{s}$. These initial conditions accommodate an elliptical LEO $(e=$ $0.20004)$ on which the tether's long-term motion is predicted to be in the form of a monotonic spin. The tangential velocity of the payloads relative to the COM at the centre of the system builds up usefully. We note that the centripetal stress is proportional to the angular velocity squared. The system responses in the time domain are given in the following Figures. Figure 3 shows a continuous build-up in the angular position of the tether over time, with Figures 4 and 5 indicating the increasing angular velocity and the tangential velocity of the tip of each sub-span relative to the spin axis located at the CoM of the tether. The increase in centripetal stress in the tether over time is shown in Figure 6 where it can be seen that this is still within reasonable limits for Spectra2000 ${ }^{\mathrm{TM}}$ at the end of the integration time of 24 hours. The orbital elements are given in Figures 7-10 with clear indication of perigee and apogee locations for $R$ in Figure 7, and a commensurate ripple in $\theta$ shown in Figure 9. 


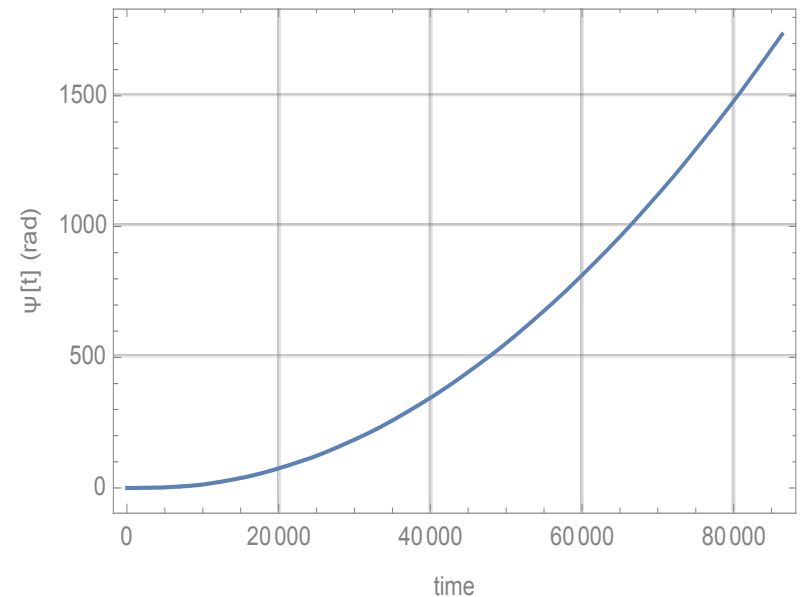

Fig. 3. Angular displacement of the tether with time.

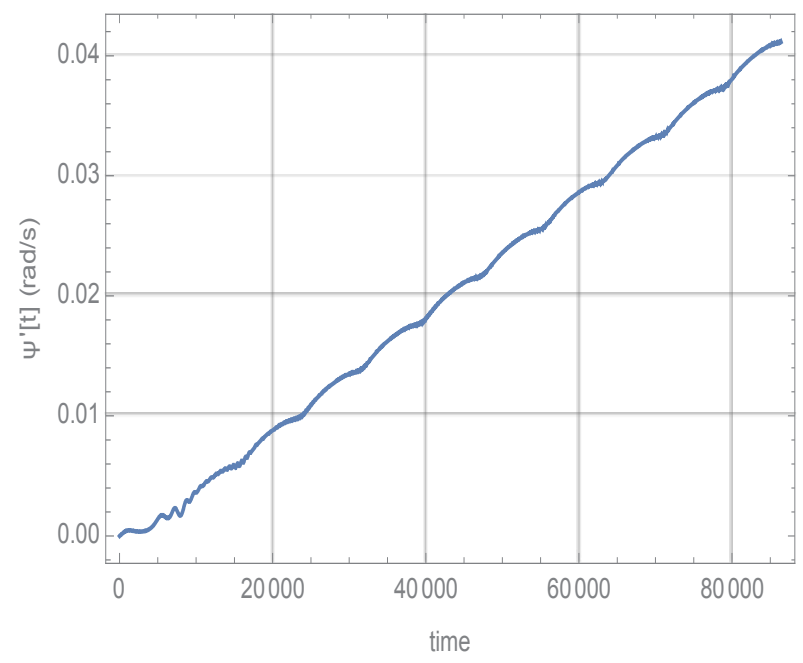

Fig. 4. Angular rate of the tether with time.

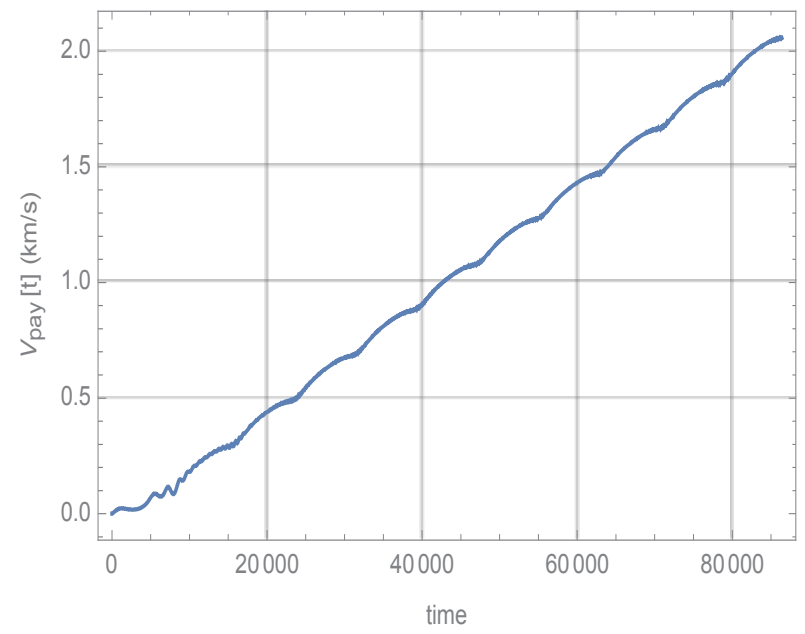

Fig. 5. Tangential velocity of the sub-spans relative to the spin axis with time.

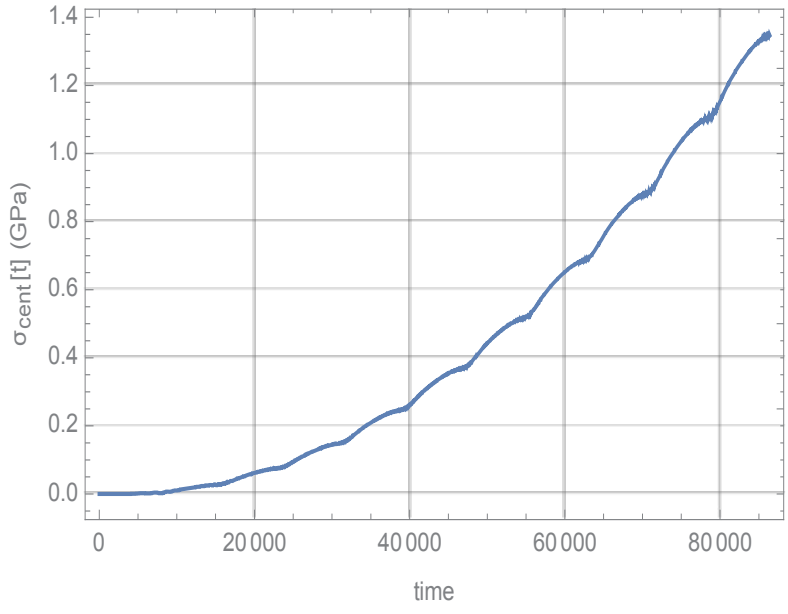

Fig. 6. Sub-span centripetal stress with time.

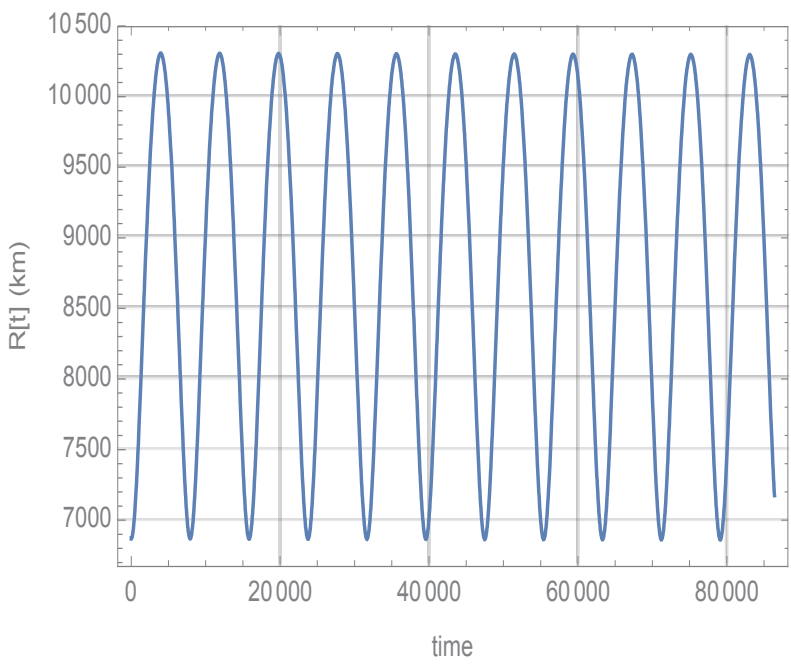

Fig. 7. Magnitude of position vector $\mathbf{R}$ with time.

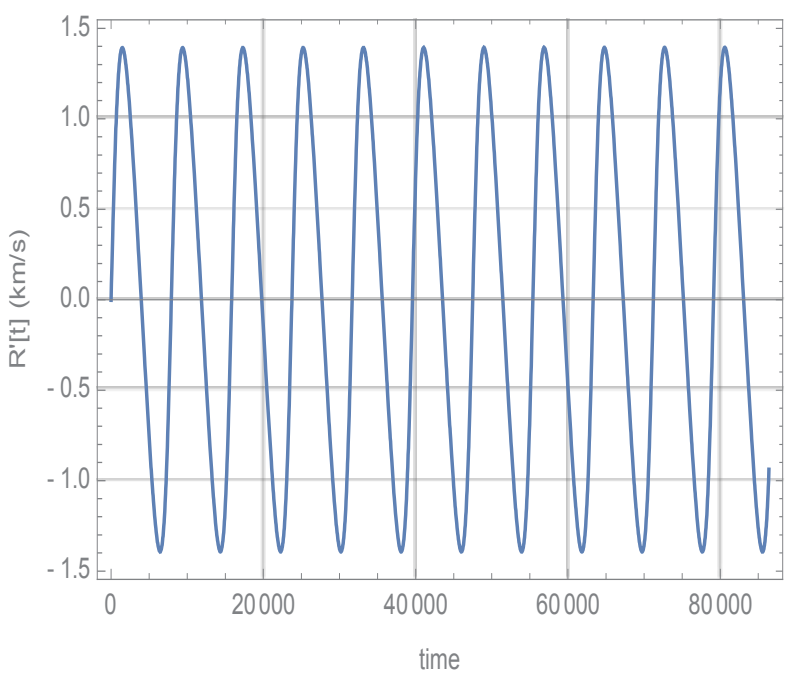

Fig. 8. Time rate of change of magnitude of $\mathbf{R}$. 


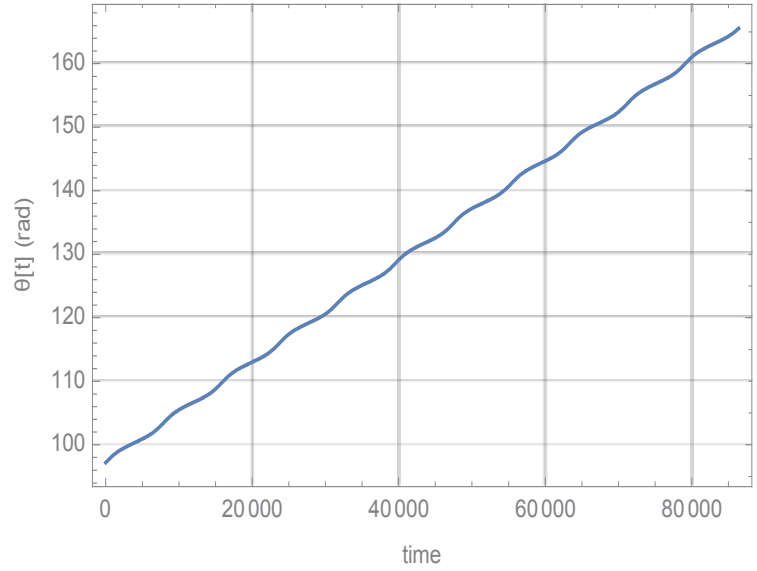

Fig. 9. True anomaly with time.

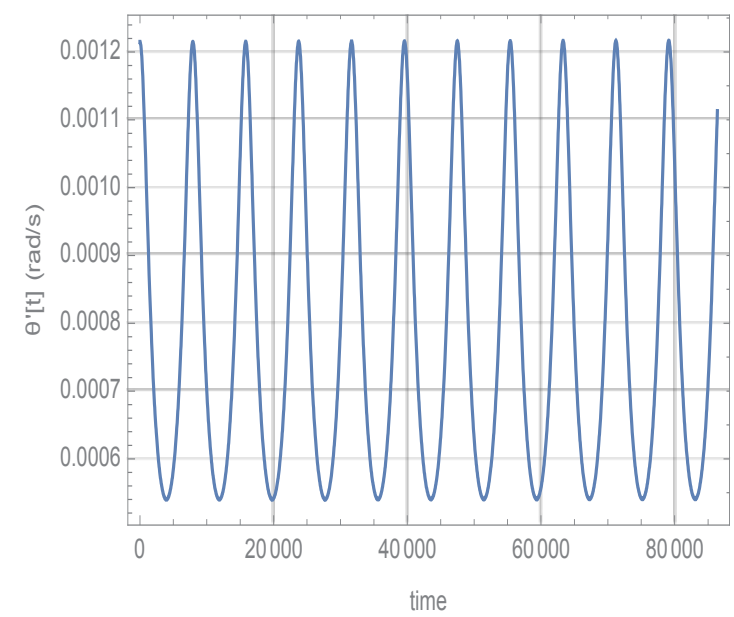

Fig. 10. Rate of change of true anomaly with time.

This model shows some interesting and typical tether phenomena on orbit and was useful for an initial data scoping study for the more complex scenario of the asymmetry case in the next section.

\section{Conditions for Mass Asymmetry}

It was shown by $[8, \mathrm{Fu}]$ that even small mass asymmetry will quickly lead to catastrophic failure of the tether as the propulsion side proceeds to collide with the outrigger counter-inertia system. A typical motorised MET system is shown in side-elevation in Figure 11.

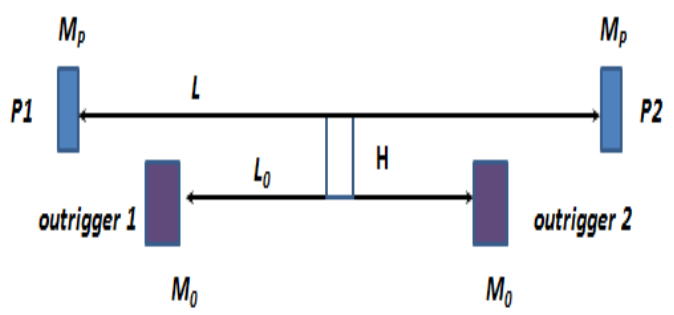

Fig. 11. Side elevation of the tether showing the propulsion and outrigger tethers and the separation distance $H$ between them.
The separation distance $H$ is assumed to comprise the height of the motor drive system. The centripetal load in a sub-span due to the mass of a payload and the tether sub-span can readily be calculated from,

$$
F_{\text {cent }}=\left(M_{p} l+\frac{\rho A l^{2}}{2}\right) \dot{\psi}^{2}
$$

and shown against time, as in Figure 12.

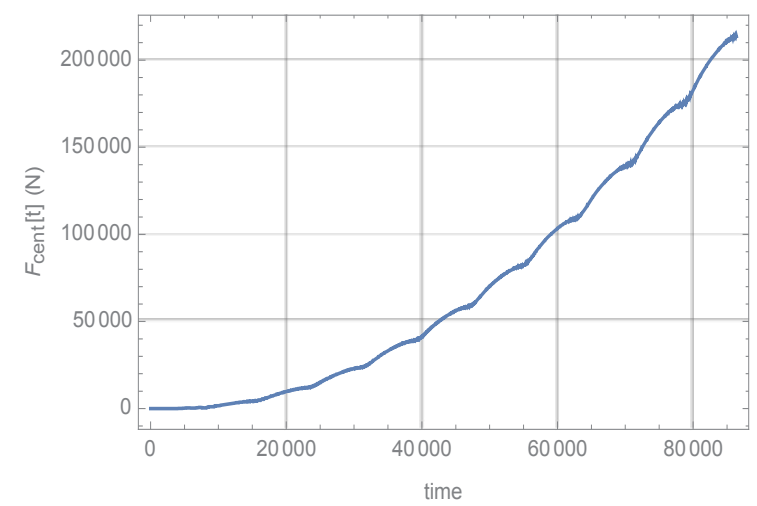

Fig. 12. Sub-span centripetal load as a function of time.

\section{Asymmetry model}

Under normal operating conditions the two sub-spans each develop equal centripetal loads, and although the moment arms, about the half-way point, of $H / 2$ mean that there are moments associated with the centripetal loads in each sub-span these are equal and opposite and therefore mutually balancing. These loads can reach $200 \mathrm{kN}$ for typical design data, and would normally be balanced by each identical sub-span. But in the asymmetrical configuration the tether will obviously be de-stabilised so that it is pushed out of the spin-andorbit plane. The precise effect depends on the physical design adopted but it cannot be ignored. For the moment arm of $H / 2$, where $H$ could be up to $3 \mathrm{~m}$, the corresponding moment could reach up to $300 \mathrm{kNm}$. A corrective counter-moment, but exerted instead over a moment arm comprising the full length of the sub-span, (taken to be $50 \mathrm{~km}$ in this example) would typically require a normal thrust of up to $6 \mathrm{~N}$ and this could easily be obtained from simple cold-gas thrusters, and provided as emergency propulsion in each payload. So, although the out of balance moment generated by asymmetry could probably be inexpensively corrected using low thrust propulsion on board the payloads there will also be the problem of whole-body translation of the tether to consider, due to subsequent re-positioning of the CoM. It is this that provides the focus of the remaining analysis, with an asymmetry scenario shown in Figure 13. 


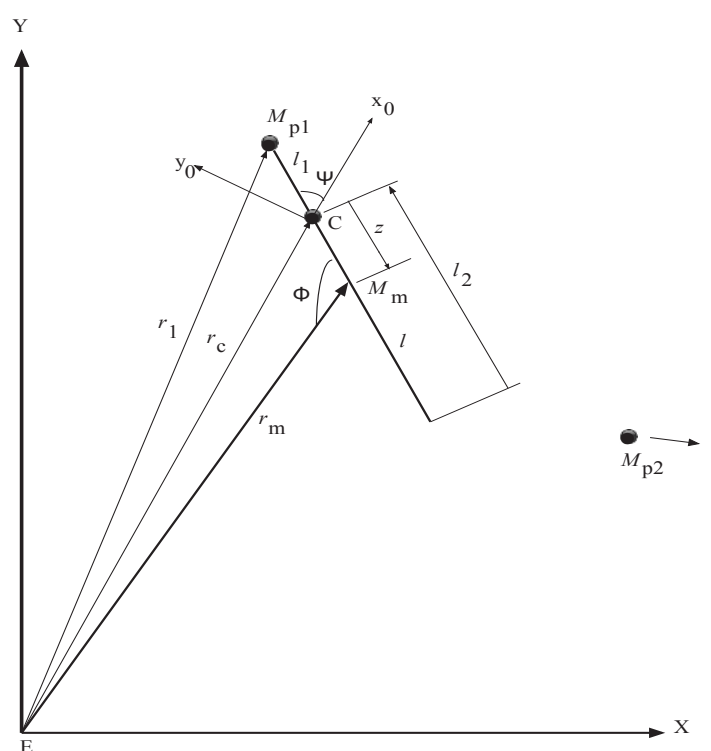

Fig. 13. Asymmetrical tether in the Earth-fixed frame with asymmetry due to the unplanned loss of payload $M_{\mathrm{p} 2}$.

The CoM is now no longer co-located with the central facility and moves closer to the remaining payload. This means that the tether will translate through $z(\mathrm{t})$ to stay on orbit. We can relate the position vector magnitudes through simple geometry to get,

$$
\begin{gathered}
r_{m}\left(-2 l r_{c} z \cos \psi+l r_{c}^{2}+2 r_{c} z^{2} \cos \psi-r_{c}^{2} z+l z^{2}-\right. \\
\left.z^{3}\right)-(l-z) r_{m}^{3}=0
\end{gathered}
$$

noting that we have dropped the arguments from $r_{\mathrm{m}}(\mathrm{t})$ and $z(\mathrm{t})$ in equations (4) and (5) for compactness. We also note that $R(\mathrm{t})$ in Figure 2 for the general elliptical orbit case is represented instead by $r_{\mathrm{c}}$ in Figure 13, where $r_{\mathrm{c}}$ is a constant radius for the circular orbit test case considered here. The procedure is to calculate $r_{\mathrm{m}}(\mathrm{t})$ in terms of $r_{\mathrm{c}}$ and $z(\mathrm{t})$ and then re-derive the equations of motion for a circular orbit condition, and in terms of generalised coordinates $\psi(\mathrm{t})$ and $z(\mathrm{t})$ to show the tether spin and also the translational movement of the tether from its initially symmetrical location on orbit. The revised kinetic energy is now given by equation (5),

$$
\begin{aligned}
T=\frac{1}{2} \dot{z}^{2}(2 A l \rho+ & \left.M_{m}+M_{p}\right) \\
& +\frac{1}{2} A \rho(l-z)\left(\dot{x}_{T l 1}^{2}+\dot{y}_{T l 1}^{2}\right) \\
& +\frac{1}{2} A \rho(l+z)\left(\dot{x}_{T l 2}^{2}+\dot{y}_{T l 2}^{2}\right) \\
& +\frac{1}{2} M_{m}\left(\dot{x}_{m}^{2}+\dot{y}_{m}^{2}\right) \\
& +\left(\frac{I_{m}}{2}+\frac{I_{p}}{2}+I_{T}\right)(\dot{\theta}+\dot{\psi})^{2} \\
& +\frac{1}{2} M_{p}\left(\dot{x}_{p 1}^{2}+\dot{y}_{p 1}^{2}\right)
\end{aligned}
$$

The potential energy remains as expressed in equation (2) except that the contribution due to $M_{\mathrm{p} 2}$ is no longer present and $r_{\mathrm{m}}(\mathrm{t})$ is re-expressed in terms of $r_{\mathrm{c}}$ and $z(\mathrm{t})$. The tether will re-balance itself by sliding along to a new CoM position on the orbit. An axial propulsive force can be introduced into the differential equation as a generalised force for $z(\mathrm{t})$ in order to counteract the displacement of the CoM. The effect of an additional $5.5 \mathrm{kN}$ axial propulsive force introduced to the asymmetrical tether, for the same numerical data as previously used, can be seen in Figure 14.

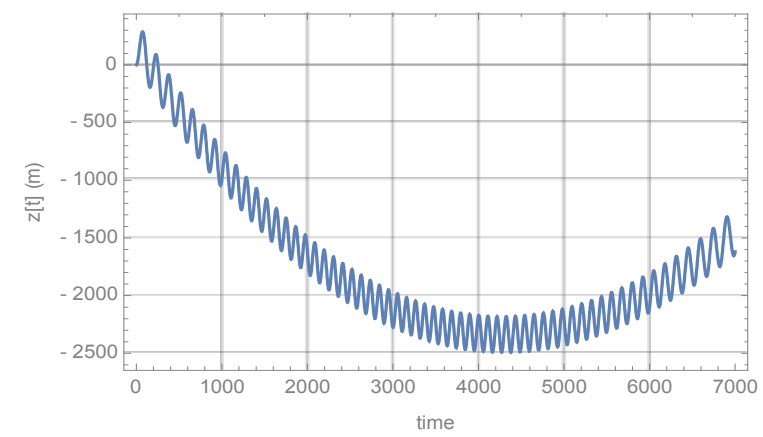

Fig. 14. Effect of axial propulsive force of $5.5 \mathrm{kN}$ on $z(\mathrm{t})$ with time.

It can be clearly seen that the whole tether starts to retract axially back from its new position from around $4000 \mathrm{~s}$, thus moving to restore the CoM to its original position over time. We also note the appearance of an axial oscillation, to which we return later. The monotonicity of the spin-up of the tether is not adversely affected but numerically the spin-up is clearly somewhat different for the specific initial conditions of the circular orbit case considered here, as shown in Figure 15.

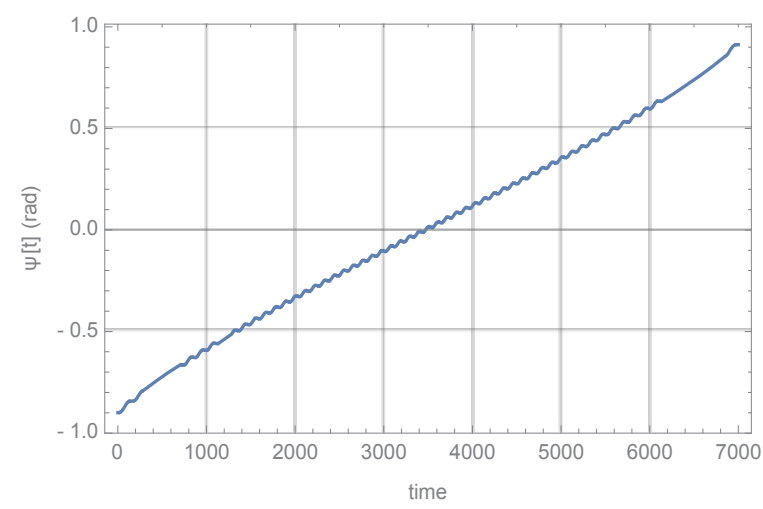

Fig. 15. Spin-up of tether on a circular orbit, with activated axial position restoration force as well as motor torque.

In summary, the tether has lost one payload, $M_{\mathrm{p} 2}$ and from that point onwards the problem has had to be treated differently - as one that is asymmetrically configured. The motor torque is applied as usual and tether spins monotonically from the initial displacement condition. It is assumed that in practice the centripetal moment unbalance is simply compensated for by a cold-gas thruster, but that the 
tether then translates so that the new $\mathrm{CoM}$ is no longer coincident with the location of the central facility $M_{\mathrm{m}}$. For the data chosen here this displacement is up to around $2.3 \mathrm{~km}$. An axial thrust, taken on observation to be $5.5 \mathrm{kN}$ in this example, is then applied (potentially from another cold gas thruster) to pull the tether back, noting that a simple uncontrolled system is shown here in which the force is simply switched on when the tether asymmetry condition arises. Obviously it will subsequently have to be reduced and then a phase of controlled thrust and counter-thrusts provided to decelerate and stabilise the tether, and also remove the axial oscillation, when it is close to its desired on-orbit configuration. This restoration force system would be highly useful for maintaining the tether spin radius so that the remaining payload can receive its expected $\Delta \mathrm{V}$, as well as for maintaining the expected configurational geometry of the tether on orbit so that the mission can continue to some extent as planned. It can be seen in Figure 16 what happens if the axial restoration force is not applied from the onset of asymmetry.

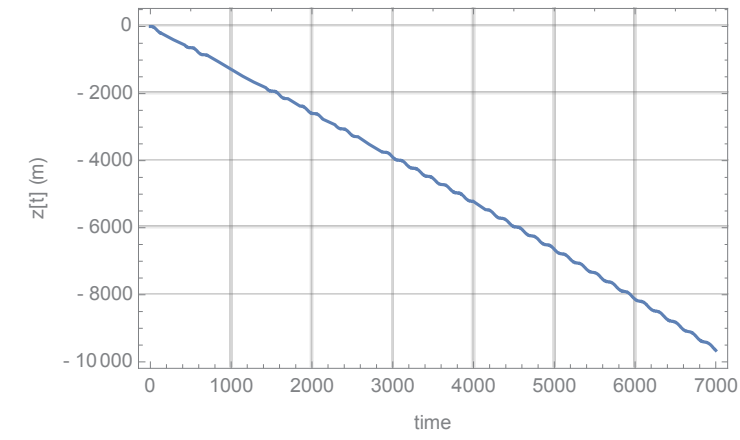

Fig. 16. The axial translation of the torqued tether with time for no axial restoration force.

It can be seen that $z(\mathrm{t})$ grows with time and eventually it can be shown that the tether no longer spins, and de-orbits. It is conjectured that all asymmetrical tether system dynamics will degenerate into some form of irregular behaviour unless a suitable controlled axial thrust is provided.

\section{Design of a Payload Transfer System for a Lunavator Tether}

A lunavator is a MMET in orbit about the moon which can be used to transfer payloads to and from the lunar surface. Payloads are captured from LEO or EEO as they enter the LSI. No previous research has considered the design of a lunavator which can both deliver and collect payloads at the lunar surface.

The system proposed herein comprises a MMET equipped with grappled payloads at either end. As the tether approaches instantaneous tidal stabilisation, the payload is extended towards the lunar surface, with the cable providing greater in-plane, and out-of-plane, positional control, and extended ground time. Crawler masses along the tether reposition to maintain lunavator dynamic stability.

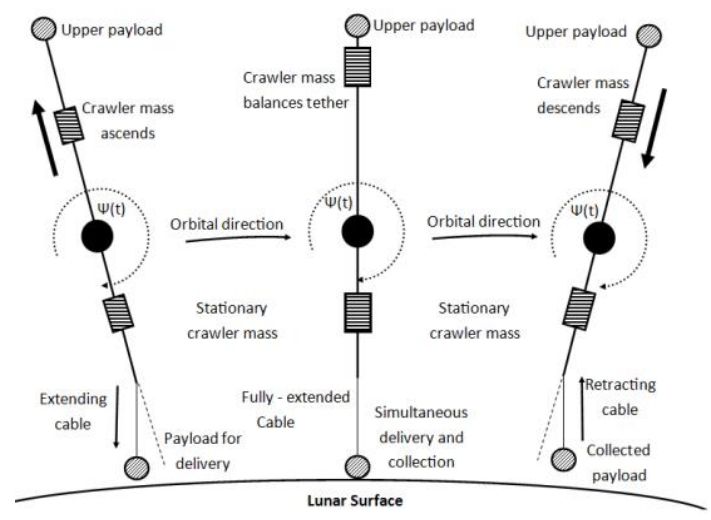

Fig. 17. Proposed lunavator design and payload transfer method

Component positions are defined relative to the orbit plane. Individual mass moments of inertia (MOI) are determined locally, and with the parallel axis theorem to the centre of mass. Due to its significant cross-sectional area, the tether is discretised when deriving its mass moment of inertia. Total kinetic, $T_{i}$ and potential, $U_{i}$ energies of each component, and propulsion braid, are also defined.

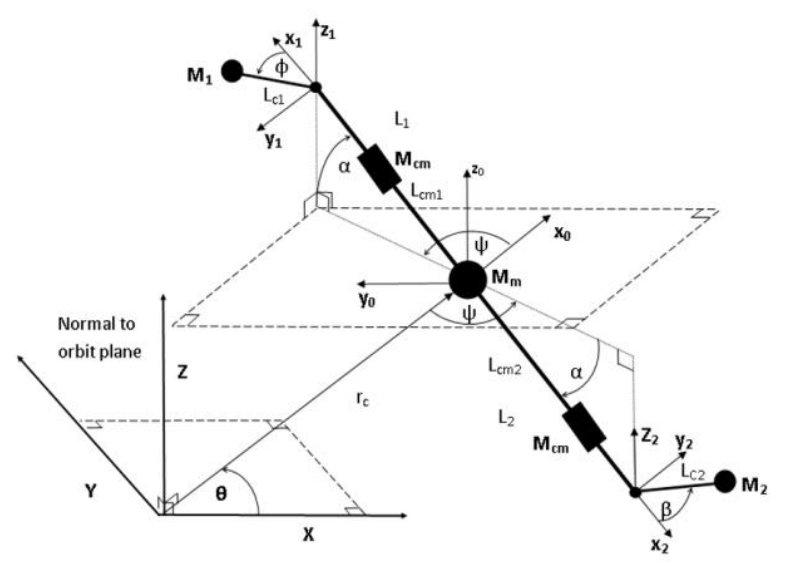

Fig. 18. 3D schematic representation of proposed lunavator

Seven representative equations of motion can be derived by substituting the potential and kinetic energies into Lagrange's Equation.

$$
\frac{d}{d t}\left[\frac{\delta T}{\delta q_{i}^{\prime}}\right]-\frac{\delta T}{\delta q_{i}}+\frac{\delta U}{\delta q_{i}}=Q_{i}
$$

where $q_{i}=\left\{L_{c 1}, L_{c 2}, \emptyset, \beta, L_{c m 1}, L_{c m 2}, \varphi\right\}$

By defining cable extension as a simple quadratic function, all seven dependent variables can be related to $\varphi$, and the model reduced to a single equation of motion for subsequent dynamic study. 
It was found that a proposed $580 \mathrm{~km}$ tether (with 2 tonne payloads) required a motor torque of $1000 \mathrm{MNm}$, with a motor mass of 1000 tonnes, to result in continuous spin (Figure 19). Smaller torque values cause in-plane tether libration wherein the lunavator cannot overcome its inertial elements (Figure 20). Alternatively, a $100 \mathrm{~km}$ tether is found to perform adequately with $25 \mathrm{MNm}$ of torque and a motor mass of 25 tonnes. Differential positioning of crawler masses may be used to ascend the tether during normal orbit, and descend during the payload transfer phases. Further work will determine the viability of such an approach.

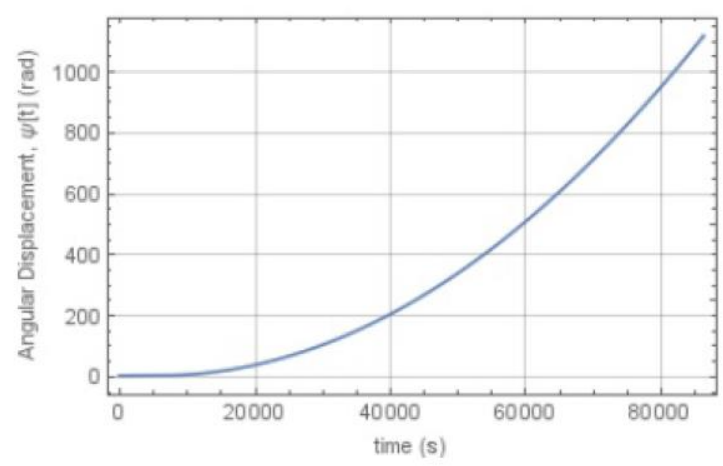

Fig. 19. Continuous spin of $580 \mathrm{~km}$ tether with $2,000 \mathrm{~kg}$ payloads, 1,000 tonne motor mass, $1000 \mathrm{MNm}$ torque

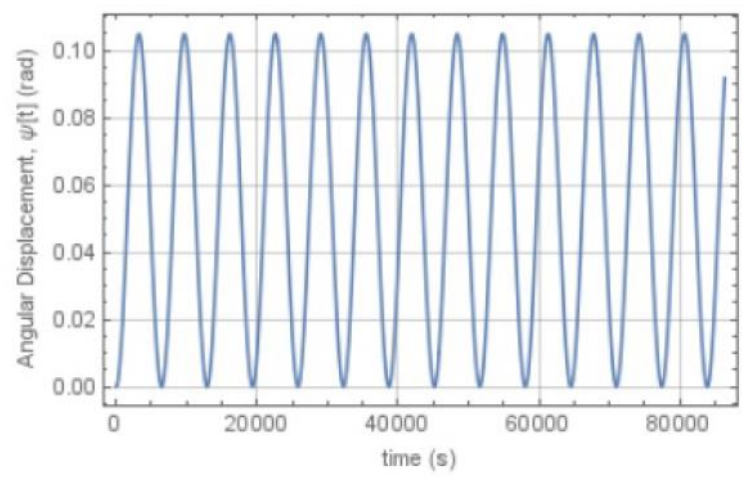

Fig. 20. In-plane libration of $580 \mathrm{~km}$ tether with $2,000 \mathrm{~kg}$ payloads, 1,000 tonne motor mass, $145 \mathrm{MNm}$ torque

\section{Deployment of a Short Tether from an Earth Orbiter for Sample Return}

Momentum exchange space tethers can be used to deorbit payloads from a vehicle travelling in a low Earth orbit. As demonstrated by the YES2 mission of 2007 [9], a payload can be swung in libration from an Earth orbiter and if released at the right moment a retrograde velocity can be transferred to it causing it to have insufficient velocity to remain in orbit, and therefore fall to the Earth.

In order to analyse accurately the requirements for deorbiting payloads of different mass and shape, a computer program was created which was capable of analysing the libration of an Earth orbiter in LEO connected to a payload via a tether. The program aimed to analyse the movements of a planar representation of a tether system comprising two masses and a solid cylinder representing a tether. This planar system is shown in Figure 21. The equation of motion for the system can be derived using the Lagrange equation as shown in Equation (6), and just as in previous sections.

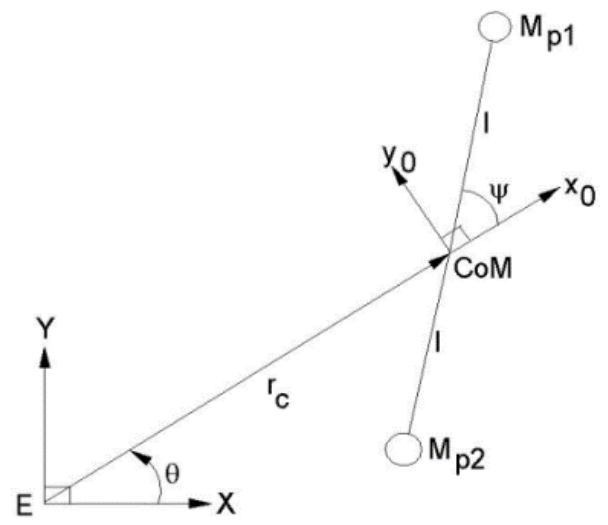

Fig. 21. Planar tether system

The equation of motion is inherently nonlinear and can be numerically integrated for different initial conditions. The angular displacement, angular velocity and tangential velocity of the payload are plotted so that the behaviour of a librating tether system in LEO can be analysed. Examples of the plots from this exercise are shown in Figures 22, 23 and 24.

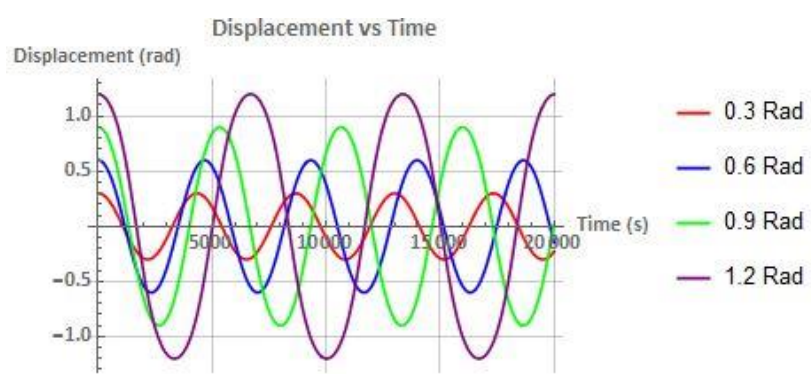

Fig. 22. An example of the angular displacement vs time chart for a tether system.

Further work is required to realise fully the deorbit of payloads using tethers. However, tethers offer a far more financially profitable and environmentally friendly solution to current payload deorbit techniques and could revolutionise payload transfer in space if suitably developed. This numerical study offers a means for the analysis of how the initial conditions of a system can effect its libration, and thus the deorbit of the payload. 


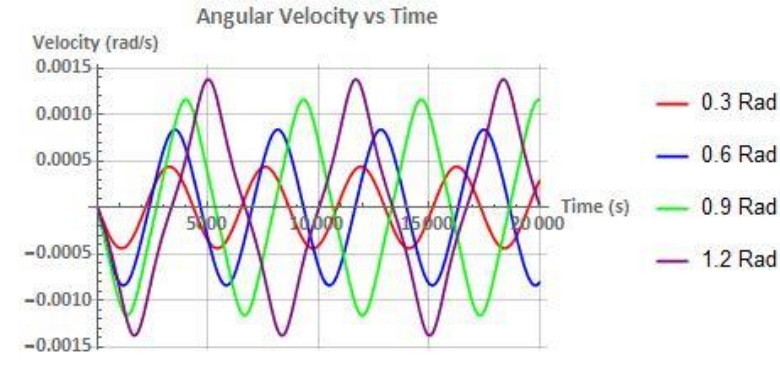

Fig. 23. An example of the angular velocity vs time chart for a tether system

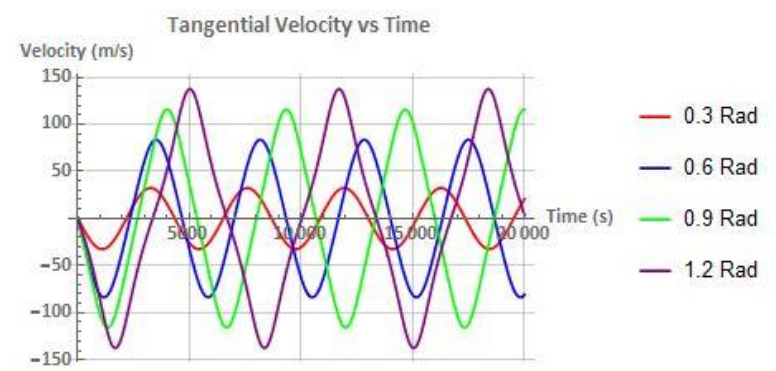

Fig. 24. An example of the tangential velocity vs time chart for a tether system.

\section{Conclusions}

Motorised tethers potentially offer clean, green, reusable propulsion in space. Many practical (equatorial and even polar) orbits could in principle be accommodated. The dynamics of space tethers are complicated, and so robust GNC and compensation propulsion will be required in all practical scenarios, particularly in asymmetrical conditions, to safeguard against failure. It may be still possible to operate a motorised momentum exchange tether on a prespecified orbit after catastrophic payload loss - if controlled axial thrust is provided by suitable emergency on-board payload propellant. METs can almost certainly be used for small sample return from an Earth orbiter. A Lunavator with reeled end-cables may offer a practical solution for smooth lunar payload delivery and retrieval. Both these applications are currently receiving further attention, with the goal of integrating them within practical mission architectures in the near future.

\section{References}

1. M.P. Cartmell, Generating Velocity Increments by means of a Spinning Motorised Tether, AIAA-98-3739, Cleveland, Ohio, USA (1998)

2. M.P. Cartmell, D.J. McKenzie, Progress in Aerospace Sciences, 44, 1-21 (2008)
3. S.W.Ziegler, The Rigid Body Dynamics of Tethers in Space, PhD Thesis, University of Glasgow (2003)

4. D.J. McKenzie, The Dynamics of Tethers and Space-Webs $\mathrm{PhD}$ Thesis, University of Glasgow (2010)

5. N.A. Ismail, The Dynamics of a Flexible Motorised Momentum Exchange Tether (MMET), PhD Thesis, University of Glasgow (2011)

6. M.P. Cartmell, C.R. McInnes, Modern Astrodynamics, Chapter 7, Elsevier Astrodynamics Series, Edited by P.Gurfil (2006)

7. C. Murray, Continuous Earth-Moon Payload Exchange using Motorised Tethers with Associated Dynamics, PhD Thesis, University of Glasgow (2011)

8. G. Fu, Research on Asymmetrical Motorised Momentum Exchange Tether, Final Research Report, Department of Mechanical Engineering, University of Sheffield, (2016)

9. Y. Chen, R. Huang, X. Ren, L. He, Y. He, History of the Tether Concept and Tether Missions: A Review, ISRN Astronomy and Astrophysics, vol. 2013, Article ID 502973, 7 pages, 2013. doi:10.1155/2013/502973 